\title{
Special Issue Robotics in Surgery and Endoscopy
}

\author{
Tobias Keck $^{\mathrm{a}}$ Ugo Boggi ${ }^{\mathrm{b}}$ Thilo Hackert ${ }^{\mathrm{c}}$ Jens Werner ${ }^{\mathrm{d}}$ \\ ${ }^{a}$ Klinik für Chirurgie, Universitätsklinikum Schleswig-Holstein, Lübeck, Germany; ${ }^{b}$ Division of General and \\ Transplant Surgery, University of Pisa, Pisa, Italy; ${ }^{~}$ Klinik für Allgemein-, Viszeral- und Transplantationschirurgie,

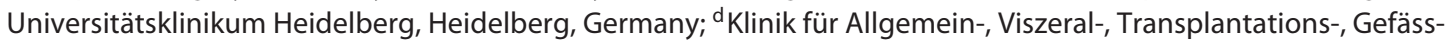 \\ und Thoraxchirurgie, LMU Klinikum, Campus Grosshadern, Munich, Germany
}

\section{Question 1: Where Do You See Robotic-Assisted Surgery 10 Years from Now? Do You See a Potential for Automatization, Image-Guided Surgery, or Automatic Navigation?}

Boggi: Robotic surgery, as currently known in visceral surgery, corresponds to da Vinci surgery. The da Vinci surgical system (Intuitive Surgical ${ }^{\circledR}$, Sunnyvale, CA, USA) is a computer-driven telemanipulator [1] and not a robot in the narrower sense of the term as it is not capable of any programmed or autonomous action. Although in most minimally invasive procedures there is still no clear evidence of the advantages of the da Vinci surgical system, users know that it does enhance surgical dexterity. In some complex procedures, such as pancreatoduodenectomy, the use of robotic assistance was associated with several advantages when compared to laparoscopy [2]. If robotic assistance becomes available at a competitive price, there will be few residual arguments against its use in most complex laparoscopic procedures.

The true revolution of robotic surgery is to have brought a computer into the operating room. We all know that evolution of computer science and technology is unstoppable, and so it will be for robotic surgery. The fact that giants such as Johnson \& Johnson and Medtronic are working on new robotic platforms confirms that robotic surgery is here to stay. But having said this, I am not sure where it will be 10 years from now. I do see a potential for automatization, image-guided surgery, and automatic navigation, but I am not able to anticipate when these improvements will be available and in which order. It is also reasonable that some improvements will be achieved more easily and earlier for some organs/procedures (e.g., navigation in fixed organs vs. automatization in pancreatoduodenectomy). What I hope for the future of robotic surgery is artificial intelligence.

Hackert: Robotic-assisted surgery will gain great importance within the next 10 years and its application will spread significantly. This trend is clearly visible in the USA as well as in Europe and China. The potential of integrating image guidance or navigation may further enhance the quality of robotic surgery as well as the expectable technical improvements of the robotic devices themselves (i.e., introduction of haptic feedback) which will be introduced in clinical routine within the next years. How far automatization may occur is unclear as the current systems are telemanipulators that do not allow automatization. The clinical reality in the next decade will probably not change substantially regarding this topic. However, the introduction of artificial intelligence and learning systems may provide such developments in a longer perspective. 
Werner: Robotic-assisted surgery will either be an established method next to laparoscopic and open surgery in visceral surgery or it will not play a role at all anymore. It will be crucial to demonstrate that there are advantages of robotic surgery compared to the already established techniques in the near future. These studies need to be performed by academic institutions and independent from industrial support. In the case that there are no clear advantages, robotic surgery will disappear due to its high costs compared to the alternative approaches.

There is potential for automatization, but this will be a slow development over the next 25 years and will probably be established in minor steps in well-defined standard procedures. Since there is no standardization in complex surgical cases, those cases will probably not be part of the automatization process. I believe that image-guided surgery and automatic navigation will be used soon and that these developments will help to provide a better quality and will support shortening the learning curve.

\section{Question 2: Robotic-Assisted Techniques Are Currently Applied in Many Fields of Visceral Medicine - Especially in Visceral Surgery. Which Application (e.g., Colorectal, Bariatric, Upper GI, HPB) Do You See as Most Promising and Why?}

Boggi: The da Vinci surgical system has introduced several technological advancements compared to standard laparoscopy. From a practical point of view, the greatest improvements of the da Vinci surgical system are the availability of wristed instruments, with seven degrees of freedom and no fulcrum effect, and the type of vision that is not just stereoscopic, high definition, and steady but also, and more importantly, immersive. EndoWrist instruments and immersive view greatly facilitate delicate dissections in narrow and deep operative fields as well as intracorporeal reconstructions. So, I believe that the advantages of robotic assistance today are more immediately evident in procedures such as esophagectomy or pancreatoduodenectomy and less obvious in purely resective procedures, such as left lateral sectionectomy or distal pancreatectomy. The importance of robotic assistance is also less obvious for procedures in which an intracorporeal stapled anastomosis is easily performed, such as in laparoscopic right hemicolectomy or sigmoidectomy, or is pursued anyway even when using the robot. It is, however, important to underscore that to enjoy the benefits of robotic assistance in complex visceral procedures, some experience has to be gained in easier operations in which the surgeon can familiarize him/herself with the system. Easier procedures can also be used as preparatory surgeries, by permitting the surgeon to practice with specific tasks before embarking upon truly com- plex operations (e.g., pyeloplasty and repair of splenic aneurysms as preparatory exercises for robotic renal transplantation). Based on this background, I believe that is important to make a clear distinction between advantages of robotic assistance in established practice (i.e., when the surgeon fully masters the system and the procedure) and the need to allocate robotic time and resources to training. As resources allocated to robotic training are expected to be paid back by improved outcomes in advanced robotic procedures, the use of the robot for educational purposes should not be seen as inappropriate.

If facilitation of intracorporeal reconstructions is clearly a strength of da Vinci systems, lack of dedicated instruments for some procedures (e.g., robotic CUSA for liver resections, robotic LigaSure for pancreatoduodenectomy) is a clear weakness of current da Vinci platforms, sometimes still making laparoscopy preferable due to availability of a wider range of auxiliary technologies.

Hackert: The future application of robotics may be beneficial in complex procedures of all fields where minimally invasive surgery is performed. Especially for rectal, upper GI, and HPB surgery, minimally invasive surgery may be facilitated, i.e., when a challenging reconstruction is part of the procedure. Despite the increasing use of robotics for procedures like cholecystectomies and hernia repair in the USA, this does not seem to be the specific field of surgery where robotic techniques will prove an advantage over conventional laparoscopy.

Werner: The most promising fields are those areas which are anatomically difficult to reach by open or laparoscopic surgery (e.g., esophageal surgery, low rectal surgery) as well as all procedures in which minimally invasive access surgery is beneficial but needs to be combined with complex reconstructions. The robotic systems allow both good access to all anatomical areas as well as precise reconstruction. I do not believe that it will be mainly applied in one field of visceral surgery only.

\section{Question 3: Education in Innovative Technologies Remains Challenging. Which Ideas Do You Have for Education in Robotic-Assisted Surgery?}

Boggi: Education in robotic surgery is a major issue. I see several hurdles that need to be solved to achieve truly structured, meaningful, and efficient training in robotic surgery.

First, considering that there are no benchmarks for most robotic procedures we miss key outcome metrics to be used as educational endpoints. In other words, even provided that we know what to teach and how to teach it, which is only partially true, we do not know when profi- 
ciency is achieved and training is completed. Should we have these reference values we could also stratify training programs in several levels and allow progression to advanced training only to surgeons who have meet benchmark values in easier procedures. Benchmark values can be surrogated from open surgery [3], but as yet we have not established the potential of robotic surgery and hence we do not have specific reference endpoints for this approach. Furthermore, since robotic surgery is hoped to improve the outcomes of open surgery, open benchmarks are expected to underestimate the value of robotic procedures. This issue is truly complex and unsolved. For instance, initial attempts at defining the learning curve for robotic pancreatoduodenectomy provided a number of 33-80 procedures $[4,5]$. However, two independent groups recently reported that results of robotic pancreatoduodenectomy are truly optimized only after $250 \mathrm{op}-$ erations $[6,7]$. Should this number become the reference point for robotic pancreatoduodenectomy, it would be extremely difficult not just to overcome the learning curve but also to find qualified teachers (even provided that all surgeons with sufficient personal experience would be willing to teach and be good teachers).

Second, training in robotic surgery comes at high costs that are not yet included in the yearly budget of both public and private institutions involved in the delivery of surgical care. Lack of dedicated resources limits the range of possible educational actions and mostly leaves the initiative in the hands of the manufacturer (that could hold private interests) or to the occasional drive of single institutions or individual surgeons. Due to financial constraints, current training programs are mostly based on short courses that are clearly not sufficient to teach robotic surgery.

Third, there is a lack of validated training pathways. All available models include a combination of theory (including machine buttonology), simulation and dry-lab exercise, observation of live surgeries, and video review, and all require a period of supervision and proctorship before implementation of independent practice [8]. Supervision and proctoring require a head-to-head relationship that makes this type of training possible but not applicable on a large scale. In addition, to be a supervisor or a proctor requires time and dedication, and is associated with costs.

Fourth, there is a need to have qualified teachers [9]. Being technically skilled and clinically proficient, alone, does not qualify as a good teacher.

The abovementioned difficulties do not mean that robotic surgery cannot be taught, but simply that we have still to make a lot of preliminary work before we can move to systematic training and exploit the full potential of robotic surgery. In my view, once all these issues are solved, the path established to teach robotic surgery will be so sound as to be used to teach all surgical procedures.
Hackert: Education plays an important role not only in the implementation of robotic surgery in a center de novo but also in teaching surgeons when using this specific method. The training should follow a distinct pathway and include in the mentioned order:

- System and simulator skills training

- Animal lab training (i.e., IRCAD) and cadaver training

- Case observation

- Proctoring on site

- Own procedure performance

If an institution is already experienced in a specific robotic procedure, case observations and proctoring can certainly be omitted and surgeons starting a robotic procedure can be supervised by the experienced colleagues in their institution; preferably, a dual-console system should be used in this setting.

Werner: The learning curve is a challenge and the systems established already are very helpful: workshops, training programs, wet labs, training consoles, and stepby-step approach to complex procedures.

\section{Question 4: Randomized Controlled Trials So Far Have Not Shown Relevant Superiority of Robotic- Assisted Techniques in Surgery in Terms of Outcome. What Is Your Opinion on This? What Are the Advantages and Disadvantages that You See of Robotic-Assisted Surgery?}

Boggi: Actually, there have been just few randomized controlled trials assessing the value of robotic assistance [10-13]. Lack of demonstration of superiority of robotic assistance in these early trials is multifactorial.

First, before comparing well-established (i.e., open surgery) and newer operations (i.e., robotic surgery), the learning curve should have been surpassed in both procedures. The results of early randomized controlled trials could have been biased by the yet incomplete proficiency of robotic assistance.

Second, most studies just compare oncologic and early outcomes of procedures. Provided that oncologic outcomes of robotic surgery must be at least equivalent to those of competing approaches to permit continued practice, early postoperative results just give a partial view of the overall value of robotic assistance. Indeed, most of these early metrics are relevant to payers (e.g., length of hospital stay), but do not provide the full picture from the patients' perspective. Later outcomes (e.g., readmissions, repeat surgery due to adhesions or repair of incisional hernia, functional results) are also important but are frequently missed.

Third, many studies were designed as noninferiority trials [10], so that superiority of robotic assistance could not be demonstrated. 
Fourth, some studies suffered from typical flaws in the design of randomized clinical trials such as focusing on too specific patient populations or choosing inappropriate treatment endpoints, thus limiting generalizability of results.

In my personal view, the advantages of robotic assistance will be eventually evident. All will revolve around proficient surgery and appropriate selection of candidates and procedures.

Hackert: The potential advantages compared to open surgery may be comparable to laparoscopic procedures less blood loss, faster recovery, and shorter hospital stay although all of this is not proven for any already established robotic procedure (i.e., prostatectomy) and only for some laparoscopic procedures. Anyway, long-term oncological outcomes are the key to show a consistent superiority (or at least noninferiority) of robotic procedures and must be investigated in randomized clinical trials. Clear disadvantages of robotic surgery are the longer operation time and the procedure-related costs (purchase of the robot and continuing expenses for instruments and single-use materials) that are problematic in countries with DRG-based reimbursement systems as long as no distinct robotic DRGs are introduced. Currently, offering and performing robotic procedures (especially complex ones) requires a considerable effort of cross-financing by the respective department or hospital.

Werner: There are no randomized controlled trials for visceral surgical procedures which support the usage of robotic surgery. Since robotic systems are now more and more frequently used for visceral surgical procedures, we will find out very soon whether there is any advantage compared to conventional open or laparoscopic approaches. The obvious advantages are a good view and a comfortable position for the surgeon during the operation. Compared to laparoscopy, the advantage is that the instruments are better and reconstruction much easier. The disadvantages are the learning curve and high costs of both the robot as well as the instruments.

\section{Question 5: Innovations Bear Chances and Risks. Do You Think Innovative Techniques Should Be Accompanied by Registries that Allow Evaluation of These Techniques? Who Should Own These Data? Should These Data Be Accessible to Industry, Societies, or the Public?}

Boggi: There is little doubt that results of innovative procedures should be prospectively recorded in registries. I also believe that these data should be audited to increase transparency and credibility, thus avoiding the risk of se- lective reporting of positive outcomes. Registries should be owned/hosted by scientific societies and, although each surgeon/center should retain the intellectual property of the data, the overall data package should be owned by societies and be regularly published with critical discussion.

Anonymized data, presented in the form of general figures, should be accessible to the public. This policy should not be specific to innovative techniques, but rather a general transparency approach used for all surgical procedures and, more in general, for all medical activities. Hopefully, public data should be stratified based on handy classes of surgical risk (e.g., age groups) so that the public can have a general idea of the risk/benefit profile in specific patient populations. Generalization of good or bad outcomes is a form of medical advertisement or alarmism that should not be permitted.

Making anonymized data available to industries appears necessary to further improve technologies, but this cannot come for free. Data could be made available to industry in exchange of support for education and research. Sharing of data must occur in accordance with local and international rules and in full respect of ethics.

Hackert: Every innovative procedure should be monitored by continuous documentation of patient and operative parameters as well as outcomes. This should be primarily done by each institution in a prospective database to identify specific potential procedure-related problems. To form larger collectives, national and international databases are certainly desirable which should be maintained by medical societies (i.e., E-MIPS). These databases should be independent from the industry in terms of financial or infrastructural support. Access to the data should be handled by a steering committee of the respective medical societies as established for other databases. Access to the data by the industry can be discussed but should be limited to clear study objectives and aims under supervision of the society responsible for the database. Public access is not recommendable due to a risk of misinterpretation and uncontrolled data spread.

Werner: It is absolutely necessary to control the implementation of new techniques by registries and by controlled trials. In addition, not only the initial implementation of the technique by some pioneers, but especially the usage of these new techniques in the general surgical community needs to be controlled to the same extent and reevaluated as well. Registries are a good tool to do so. The data of the registries should be owned by the surgeons and hospitals that report the data. The data should be reported to the medical societies. The data should also be shared with the industry to influence the development of these techniques. The public needs to be informed in an objective way. The communication to the public about advan- 
tages and disadvantages as well as indications and options for the usage of new techniques should ideally not be performed by companies, but by medical societies or national institutions of health.

\section{Participants}

Prof. Ugo Boggi, MD, FEBS

Director Division of General and Transplant Surgery

University of Pisa

Via Paradisa 2, IT-56124 Pisa (Italy)

u.boggi@med.unipi.it
Prof. Dr. Thilo Hackert

Klinik für Allgemein-, Viszeral- und Transplantationschirurgie

Universitätsklinikum Heidelberg

Im Neuenheimer Feld 110, DE-69120 Heidelberg (Germany)

thilo.hackert@med.uni-heidelberg.de

Prof. Jens Werner

Klinik für Allgemein-, Viszeral-, Transplantations-, Gefässund Thoraxchirurgie

LMU Klinikum

Campus Grosshadern, Marchioninistrasse 15, DE-81377 München (Germany)

jens.werner@med.uni-muenchen.de

\section{References}

1 Herron DM, Marohn M; SAGES-MIRA Robotic Surgery Consensus Group. A consensus document on robotic surgery. Surg Endosc. 2008 Feb;22(2):313-25.

2 Klompmaker S, van Hilst J, Wellner UF, Busch OR, Coratti A, D'Hondt M, et al.; European consortium on Minimally Invasive Pancreatic Surgery (E-MIPS). Outcomes after minimally-invasive versus open pancreatoduodenectomy: a pan-European propensity score matched study. Ann Surg. 2020 Feb; 271(2):356-63.

3 Sánchez-Velázquez P, Muller X, Malleo G, Park JS, Hwang HK, Napoli N, et al. Benchmarks in pancreatic surgery: a novel tool for unbiased outcome comparisons. Ann Surg. 2019 Aug;270(2):211-8.

4 Napoli N, Kauffmann EF, Palmeri M, Miccoli M, Costa F, Vistoli F, et al. The learning curve in robotic pancreaticoduodenectomy. Dig Surg. 2016;33(4):299-307.

5 Boone BA, Zenati M, Hogg ME, Steve J, Moser AJ, Bartlett DL, et al. Assessment of quality outcomes for robotic pancreaticoduodenectomy: identification of the learning curve. JAMA Surg. 2015 May;150(5):416-22.
6 Zureikat AH, Beane JD, Zenati MS, Al Abbas AI, Boone BA, Moser AJ, et al. 500 minimally invasive robotic pancreatoduodenectomies: one decade of optimizing performance. Ann Surg. 2019 [Epub ahead of print].

7 Shi Y, Wang W, Qiu W, Zhao S, Wang J, Weng $Y$, et al. Learning curve from 450 cases of robot-assisted pancreaticoduocectomy in a high-volume pancreatic center: optimization of operative procedure and a retrospective study. Ann Surg. 2019 [Epub ahead of print.

8 Hogg ME, Besselink MG, Clavien PA, Fingerhut A, Jeyarajah DR, Kooby DA, et al.; Minimally Invasive Pancreatic Resection Organizing Committee. Training in minimally invasive pancreatic resections: a paradigm shift away from "See one, Do one, Teach one". HPB (Oxford). 2017 Mar;19(3):234-45.

9 Gómez Ruiz M, Alfieri S, Becker T, Bergmann M, Boggi U, Collins J, et al. Expert consensus on a train-the-trainer curriculum for robotic colorectal surgery. Colorectal Dis. 2019 Aug; 21(8):903-8.
10 Illiano E, Ditonno P, Giannitsas K, De Rienzo G, Bini V, Costantini E. Robot-assisted vs laparoscopic sacrocolpopexy for high-stage pelvic organ prolapse: a randomized, prospective, single-center study. Urology. 2019 Dec; 134:116-23.

11 Jayne D, Pigazzi A, Marshall H, Croft J, Corrigan $\mathrm{N}$, Copeland J, et al. Effect of roboticassisted vs conventional laparoscopic surgery on risk of conversion to open laparotomy among patients undergoing resection for rectal cancer: the ROLARR randomized clinical trial. JAMA. 2017 Oct;318(16):1569-80.

12 Soto E, Luu TH, Liu X, Magrina JF, Wasson $\mathrm{MN}$, Einarsson JI, et al. Laparoscopy vs. Robotic Surgery for Endometriosis (LAROSE): a multicenter, randomized, controlled trial. Fertil Steril. 2017 Apr;107(4):996-1002.e3.

13 Kim MJ, Park SC, Park JW, Chang HJ, Kim DY, Nam BH, et al. Robot-assisted versus laparoscopic surgery for rectal cancer: a phase II open label prospective randomized controlled trial. Ann Surg. 2018 Feb;267(2):24351. 